Chapter 15

\title{
Laser Soldering
}

\section{Ezeonu Stella Ogochukwu}

Additional information is available at the end of the chapter

http://dx.doi.org/10.5772/51527

\section{Introduction}

The trends toward miniaturization of electronic devices and the use of expensive, temperature -sensitive components used in the telecom equipment industry have led to the demand for new, highly controllable selective laser soldering technology [1, 2]. Secondly, modern highdensity electronic and electro-optic subassemblies usually include thermally-sensitive components as well as complex three-dimensional (3-D) circuit geometries that cannot be soldered using conventional wave or reflow soldering techniques [6]. The laser is especially used for soldering of the temperature-sensitive assemblies and boards and it is also used for joining of assemblies with high thermal capacity that could not be conventionally soldered in the reflow process [2].

\subsection{Laser soldering}

Laser soldering is a technique where a precisely focused laser beam provides controlled heating of the solder alloy leading to a fast and non-destructive of an electrical joint. The process uses a controlled laser beam to transfer energy to a soldering location where the absorbed energy heats the solder until it reaches its melting temperature leading to the soldering of the contact and this completely eliminates any mechanical contact [3].

Laser soldering uses solder, which in liquid state wet the materials to be joined and provide mechanically and electrically stable connections when solidified. The ease of controlling the shape and location of the heating area leads to reliable solder joints with minimal component heating and is well suitable for high density packaging. Since it is only the solder joint region is heated during this type of soldering process without damaging the electronic components or the board, laser soldering process is of more benefit to interconnections using solders with a wide range of melting temperatures when compared with convectional infrared reflow process [4].The energy for melting of solder is applied by mean of laser beam. The use of laser 
technology provides precise heating, preventing the high sensitive components from being subjected to thermal stresses while allowing soldering of such sensitive components at high temperatures $[2,3]$. Using focusing optics allows soldering in small spaces and also of fine pitch components and the availability of motorized optics optimizes the focal point for each joint. [3].

Laser soldering has been applied in the production of electronic assemblies and in the soldering of electronics components to circuit boards [5-8]. The biomedical sector is also experimenting with the laser soldering as a means of joining tissues without sutures. Its use in this sector is driven by the desire for minimally invasive surgical joining procedure in which the surrounding tissues is unaffected [9]. A lot of work has been carried out on its biomedical applications [10-13].

\subsection{The laser soldering system}

The laser soldering system consist of laser generator, fiber optic module, focusing optics, computer vision module with camera, illuminator and the motion module, the $\mathrm{X}-\mathrm{Y}$ positioning stage with the servo control system shown in figure 1

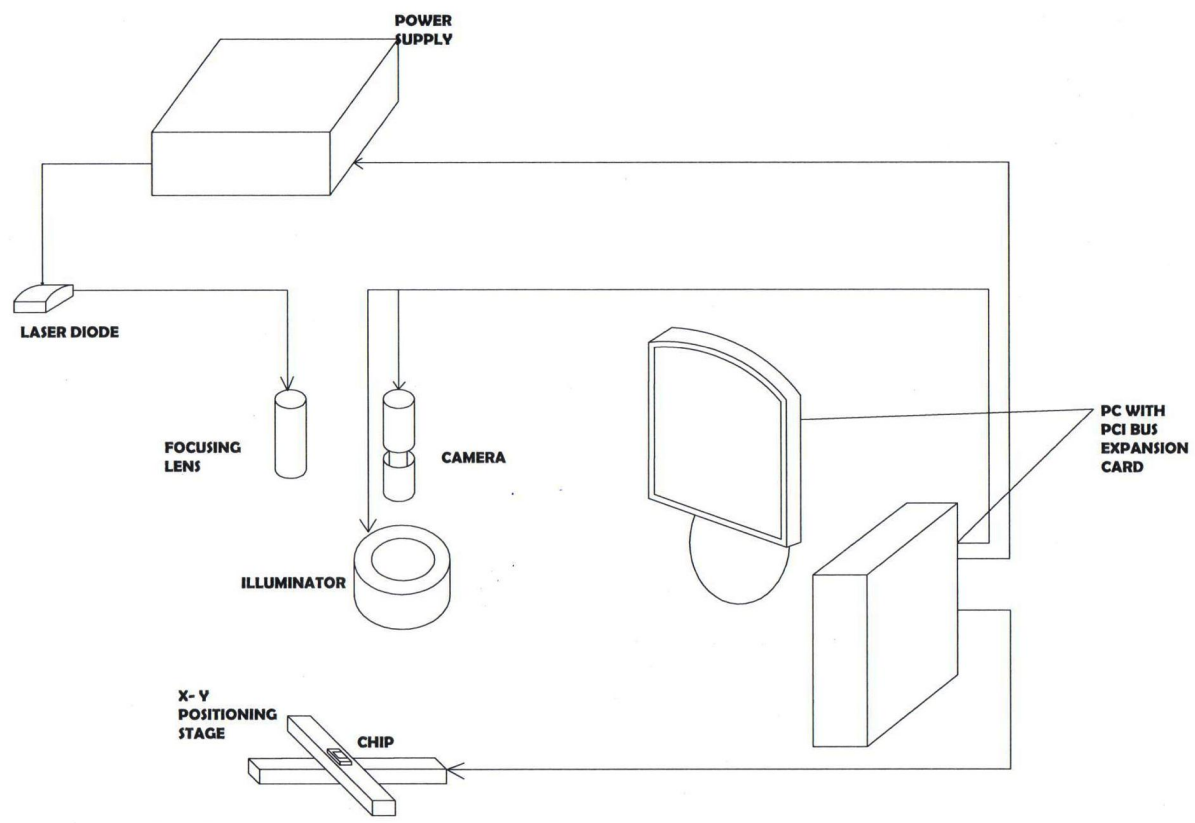

Figure 1. The block diagram of a laser soldering system

The laser beam is generated from the laser diode and modulated. With the optical system the laser beam is focused accurately on the solder joint since the laser beam is guided by a flexible 
fiber optic cable. The required temperature at the solder joint is generated by absorbing of the heat irradiation. The application of energy can be controlled precisely. This procedure is suitable both for reflow soldering with soldering paste and soldering with solder wire.

The output of the laser is delivered by the fiber optic module, consisting of input coupling optics mounted on an adjustable mount, a connectorized armor jacketed fiber cable, and the output coupling optics, which collimates and focuses the beam toward the target region.

Due to the precision and controllability offered by laser, the devices are normally mated to automated precision $X-Y$ positioning tables. The devices are placed into soldering position by a servo-operated $X Y$ table. This $X-Y$ table is used to accurately position and move the workpiece under a fixed laser beam and this actually provides precise, contactless soldering. A peripheral component interconnect-bus card in the computer controls the $\mathrm{X}-\mathrm{Y}$ stage [14]. During soldering, the movement of the laser spots on a given surface mount device (SMD) is taken care of by a computer controlled positioning mirrors, called galvanometers and these galvanometers are used to control the path of the laser. These galvanometers are programmed for each component type, and paths are stored in computer interface.

The solder material needed can be provided by wire feeding system or can be applied by solder paste.

The high level of automation of the laser soldering system results in a very repetitive process. For the process stability to be increased a closed loop temperature control of the solder joint is necessary and this can be done by using a pyrometer. This pyrometer serves as a reliable quality control during the soldering of miniaturized components in temperature sensitive surroundings [15]. The pyrometer continually monitors the surface temperature every $10 \mathrm{~ms}$ during the optimal control process and this ensures a repeatable and reliable process [16]. This pyrometer is integrated in the processing head and aligned in the optical path of the laser beam.

The CCD camera could be integrated to directly view the laser spot using accessories that allow co-axial real time viewing of the laser spot. When there is a deviation in the temperature (either high or low) of the emitted heat waves, a feedback control circuit would help in adapting the laser output in less than few seconds.

In contrast to other conventional soldering techniques, laser soldering offers a lot of advantages. They include $[6,17]$ :

- Contactless, locally limited application of energy

- Temporally and spatially well-controlled energy input,

- Low thermal stress

- It reduces intermetallic compound formation, due to rapid joint formation which results high quality joint.

- It is also has low maintenance

- Very flexible and easily adapted

- It involves a fine grain size (due to rapid cooling), resulting in better fatigue properties. 
The limitation of laser soldering lies with its extreme non-equilibrium nature. For a given laser soldering tasks, every joint have its own individual thermal mass and reflectivity and therefore demands a precisely defined laser impulse. Any slight deviation such as bent lead or a slight change in the amount of solder paste may lead to open or destroyed joint [18]. Other limitations of laser soldering include; limited ability to solder large areas, high relative price of the laser and additional cost for safety requirements.

\subsection{Process parameters}

Process parameters such as laser power, process time, and geometry of the laser beam can easily be programmed, permitting for consistent soldering results. According to [3], some variables such as the pad geometry or the size of the crown which can affect the formation of the solder joint interfere with the laser soldering process. Of importance is the ratio between the crown size and through hole. If the ratio is not optimal- if the hole is too big or too small with regard to the pin diameter, problems may occur in the reflow of tin on components sides. If the pin is either too long or too short, problems of poor adherence may occur both on the pin and pad.

In addition to physical and geometrical variables of the printed circuits and components, it is essential to take into consideration the soldering parameters applied during the process. The thermal profile control, assigned to each joint, permits the optimization of these soldering parameters particularly where the pads are connected to ground planes, the circuit has a number of layers or when the components to be soldered have a large thermal mass [3].

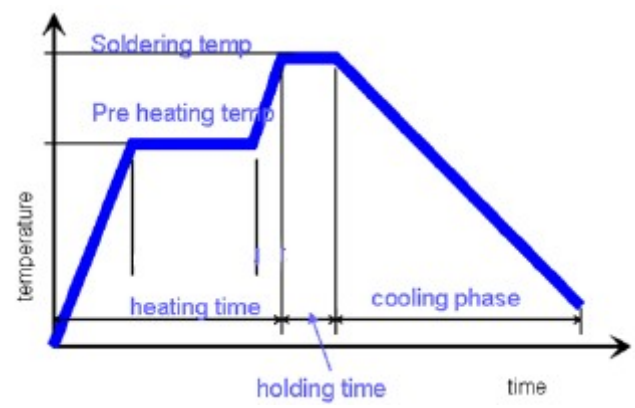

Figure 2. the soldering profile

Pyrometer used as a temperature measuring tool continually monitors the surface temperature of the joint being soldered every 10ms during the process for optimal process control [16]. Moreover, the use of pyrometer speeds up the soldering program generation process.

\subsection{Types of laser soldering processes}

There are three different laser soldering processes: single spot, simultaneous and Mask soldering $[19,20]$ : 
Single spot soldering: This method of soldering is particularly applicable for multi-dimensional configurations, tight packages and heat-sensitive components or substrates. In this process, a single contact is quickly and precisely soldered by the laser spot, offering high flexibility, quality and reproducibility. A point laser beam controlled by software moves to each programmed point. Here the solder points are heated with individually matched beam diameter and laser power profile.

Simultaneous soldering: This concept allows fast soldering of individual components or complete connection arrays. Several contact points are radiated and soldered simultaneously and no relative movement between the laser beam and the workpiece is necessary. Here lineshaped laser beams are used. it allows short cycle times with high output volume.

Mask soldering: Mask soldering is applicable for very sensitive substrates or for the soldering of fine contacts and structure. In this process, a mask is placed between the collimated laser beam which is mostly line shaped and the target. The mask covers all parts which should not be exposed to the laser radiation. The laser beam only passes through the openings in the mask only where a contact to be soldered lies underneath which ensures locally confined heating. The areas between the contact points are protected and no damage is done to the substrate. It is fast and precise.

\section{Types of lasers used in soldering}

Three main types of lasers have been found suitable for soldering process. They are carbon dioxide laser (gas laser), Nd:YAG laser (a solid state laser) and the semiconductor Laser (diode laser).

\subsection{Carbondioxide laser}

Carbon dioxide laser is a gas laser and it has a wavelength of $10.6 \mu \mathrm{m}$ in the far infrared region [21 - 23]. It is reflected from metal surfaces but is strongly absorbed by flux and the heated flux then in turn transfers heat to the solder and metal in the joint [22]. The active medium in a carbon dioxide laser is a mixture of carbon dioxide, nitrogen and helium. Carbon dioxide gas is the lasing gas while nitrogen molecules help excite the $\mathrm{CO}_{2}$ molecules and increase the efficiency of the light generation processes. The helium plays a dual role in assisting heat transfer from excited nitrogen molecules to the $\mathrm{CO}_{2}$ molecules and also helps to maintain the population inversion by getting $\mathrm{CO}_{2}$ molecules to drop from the lower laser level to the ground level [24]. The carbon dioxide laser has an efficiency of up to $20 \%$ and is cooled by pumping the gas through the heat exchanger.

\subsubsection{The pump source}

The active medium requires external pump source in order for lasing to occur. The active medium contains carbon dioxide, nitrogen and helium. The choice of pumping depends on the type of laser medium and since the active medium is in gas state, the best excitation is by 
electrical discharge of the gas. An electric discharge passing through the laser gas excites the $\mathrm{CO}_{2}$ laser. The energy of the accelerated electrons are transferred by collisions to the nitrogen molecules and then to $\mathrm{CO}_{2}$ molecules. Thus the $\mathrm{CO}_{2}$ molecules are raised to the excited state

\subsubsection{Resonator}

The design of the resonator has a very significant impact on the quality and the spatial power distribution of the emitted laser beam [25]. The carbon dioxide laser normally has a totally reflective rear mirror and a partly reflective output mirror. The cavity mirrors are often made of metal, with output coupling through a hole in the mirror rather than through a partly transmissive coating [24]. They are made with cavity optics reflective throughout the 9 to $11 \mu \mathrm{m}$ range in order to extract as much energy as possible from the $\mathrm{CO}_{2}$ laser cavity.

\subsubsection{Drawbacks of carbon dioxide laser for soldering}

There are some technological and economical reasons which prevent the use of carbon dioxide lasers as a laser source for soldering applications [6, 8, 21 - 26].

1. The energy at $10.6 \mu \mathrm{m}$ is strongly reflected by metals but undergoes about more than $90 \%$ absorption by the organic materials used for making flux, printed circuit boards and other substrates materials used for the soldering application and soldering alloys (e.g. tin-lead) have a reflective about $74 \%$ at the same wavelength. As a result, the risk of burning the circuit board is high by primary or diffused scattered laser radiation.

2. It has a high operating cost due to their electrical inefficiency (the amount of input electrical energy converted into useful laser light),

3. The $\mathrm{CO}_{2}$ laser relies on a constant gas supply and so it has to be maintained

4. The dimension of this laser source is large and the light produced by this $\mathrm{CO}_{2}$ laser cannot be delivered by an optical fiber.

\subsection{Nd: YAG laser}

This is a solid state laser. It uses Yttrium aluminum garnet doped with neodymium as a lasing substance. The neodymium is an impurity that takes the place of some yttrium atoms of roughly the same size. It emits at a wavelength of $1.06 \mu \mathrm{m}$ which is located in the near infrared region. Neodymium is an excellent lasing material as it produces the highest level of powers than any other doping element [25]. The small size of Nd:YAG rods and the optical properties of neodymium atoms limit the amount of energy that can be stored in a typical rod to about half a joule. Beam energies of 10-20 watts are normally used for soldering. Solder absorbs the thermal radiation of $1 \mu \mathrm{m}$ well which means that the light from the Nd:YAG has a high heating efficiency [23].

\subsubsection{The pump source}

The Nd:YAG being a solid state laser, has a solid crystal, and it uses light energy as the pump source. The thermal and optical properties of Nd:YAG allows it to be pumped continuously 
with an arc lamp or series of flash lamp pulses. Flash lamp pump is common for pulsed solid state lasers while continuous -wave solid state lasers can be pumped with an electric arc lamp. Energy pumping selectively energizes the $\mathrm{Nd}$ ions that subsequently lead to a cascading effect and stimulated emission of light. This light is bright enough to sustain population inversion in some solid state laser materials that are capable of continuous laser operation. The maximum average power from an Nd:YAG laser can exceed 1000W, although most operate at a much lower powers.

\subsubsection{Resonator}

The most commonly used resonator design is composed of two spherical or flat mirrors facing each other and the beam propagation properties are determined by the curvature of the reflective mirrors as well as the distance these mirrors are apart [27]. The two ends of the medium which is a solid crystal is silver coated, one slightly and the other heavily and so acts as a resonant cavity. As the laser absorbs the pump energy, the laser rod heats up. If the frequency of the pump energy exceeds the thermal relaxation time of the crystal, the temperature of the crystal increases. This induces temperature gradients in the laser rod crystal that give rise to thermal lensing, whereby the crystal acts as a lens to diffract the laser-which reduces power [25].

\subsubsection{Drawbacks of Nd:YAG laser for laser soldering}

1. Relative replacement of the light bulbs is required.

2. There is uneven energy intensity due to a decrease in the overall amount of energy delivered by the laser [21].

$\mathrm{Nd}$ :YAG laser emits at a wavelength of $1.06 \mu \mathrm{m}$ at near infrared and fortunately the near infrared spectrum is less reflective on metallic surfaces and less absorbent on organic materials thereby making Nd:YAG a more desirable candidate for laser soldering when compared to $\mathrm{CO}_{2}$ laser [21]. The shorter emission also enables flexible and cheaper beam shaping and guidance [26]. The light produced by this laser can be guided using fiber optics

\subsection{Diode lasers}

This is just like a diode, i.e. a PN-junction which is operated in forward bias mode. They are sometimes called a semiconductor diode offering wavelength ranging from 790nm to $980 \mathrm{~nm}$. A population inversion is generated in the active region by injecting electrons. By recombining electrons and holes in the active region laser operation occurs above a characteristic threshold current density. The wavelength of the laser radiation can be tuned within certain limits by varying the Al-doping of the $\mathrm{Al}_{x} \mathrm{Ga}_{1-x} \mathrm{As}$ semiconductor, and so influencing the width of the bandgap [28]. They are mainly based on gallium arsenide (GaAs) and gallium aluminum arsenide (GaAlAs).

In this diode, electrons are injected and they combined with holes. Some of their excess energy is release as photons which interact with more electrons and thereby producing more photons 
in a kind of self sustaining process called resonance. This repeated conversion of incoming electrons into photons is actually similar to the process of stimulated emission that occurs in a conventional gas-based laser.

The basic type which uses two semiconductor layers is very inefficient. Better designs have several layers which increase the power by combining more charge carriers and trapping more light inside the active layer which serves as a waveguide. The light is confined inside the waveguide where it is reflected and amplified until it exits through one end. The power of a single laser diode is low and many of it can be grouped to form a single high powered laser. When compared with $\mathrm{CO}_{2}$ and Nd:YAG lasers, diode laser offers many technological advances in laser soldering [21] and this includes

1. Due to its shorter wavelength, it has higher absorbance in metals and lower absorbance in organic materials usually used for making PCBs and other substrates.

2. It gives manufacturers a flexible and powerful tool to solve heating problems.

3. The energy density distribution of the laser beam generated remains uniform through the spot regardless of its size.

4. It is maintenance free.

5. High powered laser diode is very compact

6. The maximum conversion efficiency of transforming the input electrical efficiency of about $59 \%$, which translates into a total electrical efficiency of about $40 \%$ for a high power diode laser system and this actually caused the operating cost to be low.

\subsubsection{Pump source}

The source of pumping is by optical excitation. If a photon with more energy band gap strikes a semiconductor, it can raise an electron from the valence band to the conduction band, creating an electro-hole pair, a pair of current carriers. This effect can be used to detect light by generating an electric current proportional to the amount of illumination. The current passing through the semiconductor laser produces a population inversion in the junction which is incidentally the active layer. Inside the junction, the composition of the semiconductor changes. Population inversion in this case would be a large concentration of free electrons and holes in the same region.

\subsubsection{Resonator}

In conventional laser system, a laser beam is produced by pumping the light emitted from the atoms severally between two mirrors. In a laser diode, an equivalent process occurs when the photon bounces back and forth in the Junction (the active layer) between the N-type and Ptype semiconductor. This type of resonator is known as Fabry -Perot resonant cavity. The two parallel polished planes are used to produce multiple reflections in the cavity in order to achieve a very high intensity of laser beam. The amplified laser light eventually emerges from the polished end of the cavity. 


\section{Diode laser soldering}

The diode laser is a semiconductor device that directly converts electrical energy into laser light. Mostly, higher power diode laser output in the near infrared, at either $808 \mathrm{~nm}$ or $980 \mathrm{~nm}$. The size of diode laser is small and this makes them easier to be integrated into workstations. They produce their waste in a relatively small physical area and as a result, can be cooled with a small volume of circulating water and a chiller.

In diode laser soldering, a focused laser beam provides fast and controlled heating of the solder alloy to reflow the solder and form stable joints. Lasers with output power in the range of 30W-80W are sufficient for soldering applications [29].

A typical diode laser soldering system consists of a laser/control unit and a fiber cable is used to deliver the laser spot to any desired location. The laser/control unit provides diode laser temperature and current control and the output can be pulsed using internal and external system interfaces and this makes them easily adaptable to automation.

Higher power diode laser soldering has been adopted by some electronics and telecommunication equipment manufacturers because of the following reasons [2, 29];

1. It provides temporal and spatial process control and this control extends to both the location and the metallurgy of connections, resulting in optimized joints for thermally sensitive components.

2. Automated solutions for complex applications - the complex three dimensional (3-D) circuit geometries with thermally sensitive or high value components. Difficult - to -reach locations and fine - pitch quad flat packs

3. Easily tailored to specific joint design and consistent high quality joints.

\subsection{The process of a diode laser soldering}

The laser beam is generated from the laser diode and is focused precisely on the solder joint with the use of an optical system. The required temperature at the solder joint is generated by absorption. This method is suitable for both reflow soldering with paste and soldering with solder wire.

For selective reflow soldering, the soldering paste is dispensed first, then warmed slowly and the solder joint is preheated. Finally the soldering paste is melted totally, a meniscus is then formed at the solder joint and the contact is covered by the solder completely.

For soldering with solder wire, the process occurs in three steps [30]

\section{Step 1: Pre-heating}

The laser is switched on and the solder wire enters the range of the laser beam. The direct radiating heats the wire up close to melting temperature. If the laser beam hits the PCB outside the solder pad the surface of the PCB may get burned.

\section{Step 2: solderwire feeding}


This process is important for an accurate soldering process. The solder wire is fed during the solder feeding time at a specific speed. The solderwire with temperature close to melting temperature hits the solder spot and melts at the preheated solder pad and the preheated pin. If the solder pad and pin are not warm enough, the wire gets burred or bends. The solder wire feeder is driven by a DC-Motor and an encoder is used to control the feeding rate. The feeding length and feeding rate are programmable.

\section{Step 3: Hold time}

The solder wire having melted, can spread evenly to build the typical shape of a solder spot. Liquid solder has a significant lower absorption rate. It acts like a mirror. In this phase of the soldering process the laser beam can be partially deflected and can damage possible surrounding components or the plastic housing.

The beams of many single diodes must be bundled to the focus point in order to have the energy of approximately $30 \mathrm{~W}$ to a focus point of $0.8 \mathrm{~mm}$ diameter the and this is only possible with complex optics (see figure 3). The beam of the laser diodes is at first collimated in one axis by a cylinder lens and is then coupled into the optical fiber.

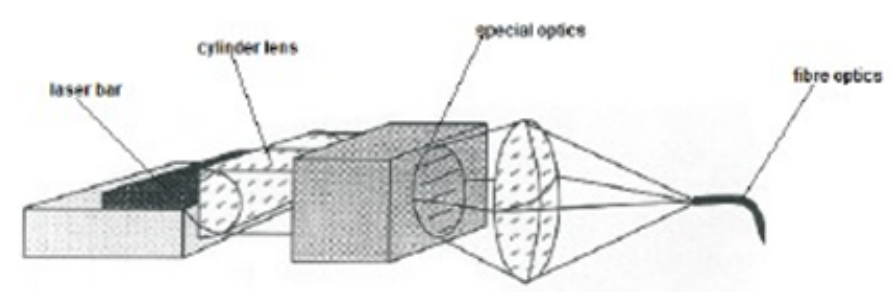

Figure 3. Laser diode with the optics delivery beam. Adapted from [30]

\subsection{Soldering with high power diode lasers}

Hoult [2] stated that in the microelectronics industry, for soldering tasks to be done, an average laser power of 2 to 80 watts can used. This laser average power depends on solder joint dimensions and the required speed. The soldering tasks are categorized by size into small, medium and large soldering areas [2].

\section{Small, 40 to $100 \mu \mathrm{m}$ (0.0016 to 0.004 in.) Pads}

Typical applications for soldering these small pads are in high-density packaging. A few watts of average power are normally sufficient to solder these joints. Specific spot size and working distance requirements can be addressed by a host of commercially available optical imaging accessories (OIAs).

\section{Medium, 100 to $500 \mu \mathrm{m}$ (0.004 to 0.02 in.) Pads}

In these cases, 25 watts delivered from an $800-\mu$ m-diameter optical fiber with an OIA capable of reducing the source size by a factor of 1.8:1 offers some benefits. Dwell time is approximately 1 second per solder joint. 


\section{Large, 1 to $3 \mathrm{~mm}(0.04$ to 0.12 in.) Pads}

In some cases, it is very essential to scan several joints simultaneously and this can be done by expanding the spot size of the diode laser. This technique is a viable solution for soldering multiple joints on a densely populated printed circuit board (PCB). To increase the scan time, higher average power diode laser systems (up to 80 watts continuous wave) can be employed.

\subsection{Laser soldering parameters}

Hoult [2] has also identified a list of laser soldering parameters to be considered while developing a soldering process in order to achieve a high quality joint. They include the following

1. Average power: The average power of the laser controls the rate of heat delivered to the joint being soldered. High average power is preferred since it reduces the soldering time, but the excess power causes vaporization and also reduces the quality of the joint.

2. Pulse time/length: Just like average power, pulse time/length controls the amount of energy delivered to the joint by a laser.

3. Pulse duty cycle: This modifies the rate at which heat is delivered to the joint thereby increasing the control of the soldering process. A high duty cycle, because it permits a minimum soldering times, is therefore preferred.

4. Laser power density (intensity): The laser power density (intensity) controls the response of the material to the laser beam and in association with the average power, generally determines the rate of the soldering process.

5. Laser focus position: The accurate placement of the laser focus spot is very critical in order to secure a good quality joint. This is best achieved by using precision $\mathrm{x}-\mathrm{y}$ positioning tables coupled with a CCD camera and an imaging accessory that allows viewing of the laser beam in real time.

\section{Laser soldering as a selective soldering process}

Years back, most of the components in all electronic products are through-hole (TH) components but today about $90 \%$ of the through -hole components in these electronic products are being replaced by their surface mount counterparts [1]. This may be to the benefits of the surface mount technology (SMT) with regard to through-hole technology (THT) and they include [31]:

- Increased circuit density

- Lower costs in volume application

- The reduction in the size of the board

- The reduction in the size of the components 
- Shorter pins

- Shorter interconnections

- Facilitated automation

- Improved electrical performance.

Though many through-hole components are being replaced by their surface mount counterparts, printed circuit boards (PCBs) are still being designed with both technologies. This is because despite the benefits of SMT in relation to THT, the through-hole components will remain in electronic industry for many years because of the [1]:

- Component availability

- Solder joint reliability

- The need for socketability.

The hot air and convection reflow soldering methods are primarily used for SMT components. The hot air is used for low volume application while the convection reflow is used for high volume application. Hand and wave soldering are primarily used for TH components, though a few SMT components can be soldered by these methods.

When confronted with mixed assembly board, there is always a challenge to determine the optimal method that can be used to solder these boards, and then selective soldering becomes the answer.

In selective soldering, only the through-hole components are selectively soldered after the surface mount components have been reflow-soldered. Therefore selective soldering is defined as a process of soldering only through-hole components onto a printed circuit board (PCB) that has surface mount components on the underside.

In a mixed- assembly board, through-hole components can be selectively soldered by three methods [1].

Wave soldering, with or without specially designed fixtures: This can be used for throughhole whenever the board's secondary side contains devices that can go through the wave solder process. The through-hole components must be selectively soldered without impact to the adjacent components already soldered in the reflow oven. Depending on the application, selective wave soldering using text fixtures can be very expensive.

Convection reflow using paste-in-process: This allows reflowing of both the through-hole and surface mount device (SMD) at the same time but the difference and distribution of thermal masses on circuits may lead to different results in terms of the quality for different components. When the component is temperature-sensitive, has too many rows of pins to allow sufficient solder paste deposition, or the board is not designed for the paste-in-hole process to begin with, the paste-in-hole process using convection reflow cannot be used [1].

Hand soldering: This has always been the backbone of electronics assembly for decades. They can be used to complete connections that are not possible using other methods. The compo- 
nents that cannot withstand the high temperature involved in the wave soldering process are soldered by hand. This method is not only slow and expensive but unfortunately the quality of the joint being soldered depends on operator's capability.

For cost effective selective soldering of through-hole components when either wave, hand or convection reflow is either not technically possible, desirable or too expensive, laser soldering comes into play. Laser soldering does not need any special fixture and is capable of meeting the requirements of mixed SMT and TH fabrication in terms of the quality, repeatability aswell as the flexibility [1]. The miniaturization and integration of electronic components and the use of temperature-sensitive components used in telecommunication equipments also contributed to the rapid growth of this field.

Laser soldering is a desirable process used to solder areas that are difficult to reach or parts which cannot be soldered by either wave or reflow soldering. These can be parts that are heat/ temperature sensitive and is repeatable [22] or need a special temperature program due to their size. The power control of the laser source and the steadiness of the output power are necessary to guarantee a steady repeatable process [3]. The use of optics developed for the soldering process produced conically shaped beams and this allows it to be moved and focused on a solder joint. The use of laser technology for selective soldering permits precise heating, thereby avoiding the subjection of high thermal stress on the heat-sensitive components at high temperatures.

The benefits of a selective soldering includes but not limited to [33]

1. It allows the user to optimize the solder process down to pin level verses the compromise techniques in flux application.

2. It improves the quality of the solder and reduces the thermal coefficient of expansion issues with minimalistic surface mount components that do not tolerate the thermal shock of wave soldering.

3. Selective soldering is ideal for thermally challenged through-hole components since the flux deposition solder dwell time and peel-off parameters are fully programmable.

4. It is used to solder printed circuit board assembly (PCBAs) with high component density since it can maintain clearances between the through-hole pads and adjacent surface mount pads that are not achievable with masking pallets and permits the soldering of through-hole components on both sides of the PCBAs without restricting the components height.

5. It offers the ability to reduce solder defects, hence improving the first pass yields.

\section{Comparison of laser soldering with most of the common soldering methods}

Laser soldering is mainly characterized by short duration heating and high intensity radiation which can be focused on a very small spot. There are other types of soldering techniques - Iron 
soldering, wave soldering, induction soldering etc. They also offer a lot of advantages and disadvantages but when compared to laser soldering, they have the following characteristics;

1. Iron Soldering

- Contact heating

- It requires high maintenance since the tips wear with use and has to be replaced periodically.

- Inexpensive

- Iron's heat spreads beyond the joint area and so the sensitive components in close proximity may be destroyed.

- Regular cleaning of the tip to remove flux and oxide build up

- Long processing/soldering time

2. Induction Soldering

- Soldering from underneath - not above

- It has high energy capacity -Ability to melt high temperature solders

- Complexity of determining appropriate settings based on material conductivity

- Nitrogen atmosphere is necessary.

- Appropriate for large scale productiion

- Flux application is necessary

3. Wave Soldering

- The need for nitrogen atmosphere is necessary

- Low cost

- Separate preheating and flux application is necessary

- Soldering is from underneath

- Difficult to use with diverse package types especially ball types SMD packages and narrow lead pitch SMD packages.

- High thermal stress for SMD but low for THD.

- Appropriate for mass production

4. Infrared Soldering

- High thermal stress.

- Difficult to heat components that are in the shadows.

- Uneven heating (temperature variations) arise in places being soldered due to component shapes. 
- Low cost

- Separate preheating and flux application is necessary

5. Convection Reflow Soldering

- Nitrogen atmosphere is necessary

- Separate preheating and high demand for flux activity

- High thermal stress

- Easy heating of components that are in the shadows

- Uniform heating across product is possible i.e. even temperature distribution

- Long processing time.

6. Vapour Phase Soldering

- Separate preheating and inert liquid atmosphere is necessary

- No temperature control system is required

- High thermal stress

- Minimal oxidation and contamination of soldered areas

- Appropriate for mass production.

- Even heating is achieved regardless of the shape of the components

7. Resistance Soldering

- Contact heating

- Heat may not reach the right spots due to temperature variations in contact resistance

\section{Industrial applications of laser soldering}

Industrial lasers deliver large amounts of heat with great precision and without contact, making them ideal for applications such as soldering. Laser soldering uses the well-focused, highly controlled beam to deliver energy to a desired location for a precisely measured length of time. Laser soldering is applied in the following areas.

\subsection{Photovoltaic module manufacturing}

In photovoltaic module production, solar cells get interconnected by strings which are then laminated in modules. The solar cells are stressed thermally and mechanically during cell soldering and string handling. As solar cells are currently becoming thinner and thinner and thus more fragile, soldering and handling is becoming more difficult. Due to the trend in the decreasing thickness of the solar cell $(<200 \mu \mathrm{m})[34,35]$, the demands for gentle production 
methods to reduce breaking of the silicon wafer during manufacturing and soldering method without any mechanical contact has led to the application of laser soldering in photovoltaic module production.

The string handling can be avoided completely by laser soldering directly on the laminate layers. This method is called In-Laminate-Laser-soldering (ILL). Laser-soldering is a connection method that does not induce mechanical loads to the solar cells and reduces thermal stress on the cell. The laser beam produces mechanically and electrically stable solder joints between the connector and the solar cell in fractions of a second. A high power diode is used because it has all the properties for contacting thin film solar cell [35]. It uses a non contact technology with a precise and locally limited thermal input.

The pyrometer sensor integrated in the processing head and aligned in the optical path of the laser beam is used to control the process.

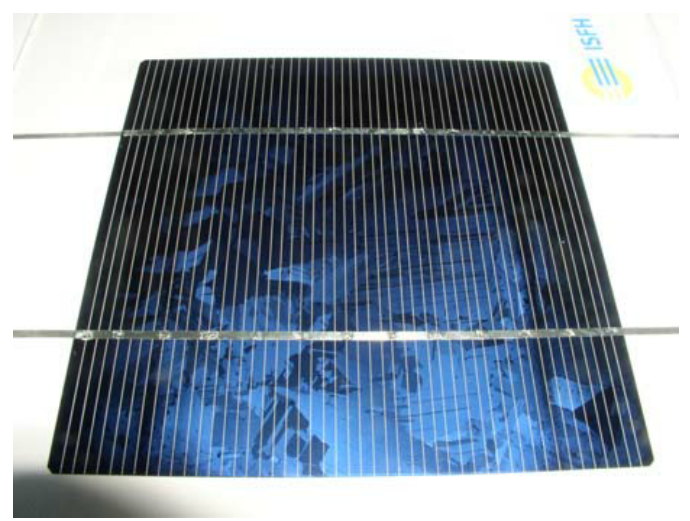

Figure 4. With In-Laminate Laser Soldering (ILLS) manufactured solar cell module. Adapted from [35]

\subsection{Electronic manufacturing}

The automation in the telecommunication equipment industry, miniaturization of electronic devices in consumer electronics and other biomedical applications which produces high density microelectronics with fine-pitch leads and small pad diameters has led to the demand for highly controllable selective laser soldering. This is because these devices often have the complex three dimensional (3-D) circuit geometries with thermally sensitive or high value components such as sensors, lenses central processing units etc that cannot be soldered with conventional wave soldering techniques.

Laser soldering has a lot of attributes but the main attributes are short duration heating and high intensity radiation which can be focused on a spot as small as $0.050 \mathrm{~mm}$ in diameter [31] and this is beneficial to soldering densely packed regions, where local solder joints can be made without affecting the nearby components. 
Nd:YAG lasers and carbondioxide lasers have been successfully applied in industrial production but the development of high power diode lasers recently has offered a new laser source for soldering with technological advantages. The absorptivity of laser radiation on metals, generally increases with shorter wavelength and, consequently, diode lasers may lead to a higher process efficiency compared to Nd:YAG lasers and carbon dioxide lasers [21, 36]. The high power diode laser has been adopted by some electronics and telecom equipment manufacturers because it offers high reliability, ease of automation and temporal and spatial process control [2]. This control extends to both the location and the metallurgy of connections, resulting in optimized joints for thermally sensitive components, special substrates and some areas difficult to reach [6]. Selective laser soldering permits the delivery of precise amount of energy to specific locations without causing heat related damage to the surrounding components.

\subsection{Automobile application}

In automobiles, Laser soldering offers a better and perfect alternative to conventional techniques due to the increasing number of electrical contacts in combination with more complex and miniaturized components [36].

Laser application for soldering permits highly automated production system, providing enormous flexibility for a wide variety of fabrication tasks at a very high processing speed because it is a non-contact technique. The mating parts are joined by a solder and the melting point of the solder is usually lower than that of the components materials. When the solder is melted, it flows into the gap between the parts and bonds with the surface of the workpiece. The thin gap between the components provides the capillary function, thereby drawing the liquid solder into the joint.

The surface of the solder seam is smooth and clean and normally requires no refinishing; they are often used in automobile industry for making body parts such as car roofs or trunk lids.

\subsection{Medical application}

The desire for minimal invasive surgical joining procedure led to the use of laser soldering in medical applications and this is because for a fast healing process, a successful joining of tissue is very important. Laser tissue soldering is the process of using laser energy to join tissues without sutures and it is based on some soldering material ( such as albumin proteins - a biomedical solder) that coagulates on interaction with a laser beam to form joint [9].

Laser tissue soldering has been successfully utilized in bonding different type of tissues, including the cartilage \& blood vessels [10], skin [11], liver [13]..

Diode lasers can be used to repair without damaging the surrounding tissues. This technique shortens the surgical times and helps minimized trauma to tissue speeds healing [29].

Laser soldering of biological tissue is a unique and promising technique because of the following reasons [29];

- No damage is done to the surrounding tissues 
- Precise targeting

- Highly reliable

- Compact size and portability.

Laser tissue soldering is gaining importance because the laser technology actually fulfills the high quality requirements to perfect hygienic surfaces, dross free and free of any material residues.

\section{Conclusion}

Laser soldering provides a clean non-contact process which involves transferring energy to the soldering location using a precisely controlled beam. The laser beam is mainly absorbed by the solder leading to a fast and high quality solder joint. The very short heating and cooling times leads to a fine-grained structure of inter metallic bond. Due to its precise and contactfree process, laser soldering produces the best quality of solder joint

As a selective soldering process, it enables the precise amount of energy to be delivered to specific soldering areas, even those areas difficult to reach, without causing collateral heatrelated damage. It represents the best method to solder surface mount components onto the printed circuit boards.

\section{Author details}

\section{Ezeonu Stella Ogochukwu*}

Address all correspondence to: ogooezeonu@yahoo.co.uk

Department of Physics/Industrial Physics, Nnamdi, Azikiwe University, Awka, Nigeria

\section{References}

[1] Prasad, R P. (2004). Laser soldering: Technology of Today and Tomorrow. SMT magazine, April 2004, 18 (4) : 14 http://www.smtonline.com/pages/zone.cgi?a=64726,Accessed 2012 April 25.

[2] Hoult, T. (2004). Laser Solutions for Soldering. Circuit Assembly.j, http://www.coherent.com/Downloads/circuitsassembly0204Rev2.pdf.,Accessed 2012 March 30.

[3] Raga, A, \& Carlson, K. (2011). Selective Soldering by Laser. http://www.us-tech.com/ Relld/.../selective_soldering_by_laser.htm, Accessed 2012 May 15. 
[4] Messler R. W \& Millard D. L (1994). Laser Soldering: New Light on an Old Joining Process. Welding J , 73(10), 43-48.

[5] Semerad E., Musiejovsky, L., and Nicolis, J. (1993). Laser Soldering of SurfaceMounted devices for high Reliability Applications. J of Material Sci. 28: 5065-5069. Avalilable: www.springerlink.com/index/pdf.Accessed: 2013 March1

[6] Beckett, P. M, Fleming, A. R, \& Foster, R. J. Gilbert, J.M \& Whitehead D.G ((1995). The Application of Semiconductor Diode Lasers to the Soldering of Electronic Components. Optical and Quantum Electronics Available: www.springerlink.com/index/ r140g33338565063.pdf.Accessed 2013 Feb 24, 27, 1303-1311.

[7] Lea, C. (1987). Laser Soldering of Surface Mounted Assemblies. Microelectronics Int. Avaliable: www.emeraldinsight.com/journals.htm?articleid=1666621.Accessed: 2013 March 9, 4(1), 36-42.

[8] Beckett, P. M, \& Fleming, A. R. Gilbert, J.M \& Whitehead D.G ((1997). Practical Application of Laser Soldering in Electronics Assembly. Proceedings of IEEE Int. Symposium on Ind. Electronics. ISIE'97. Avaliable: ieeexplore.ieee.org/ iel4/5230/14218/00651791.pdf. Accessed: 2013 Feb 24, 1, 59-64.

[9] Ion, J. (2005). Laser Processing of Engineering Materials; Principles, Procedures and Industrial Applications. 335 p.

[10] Ott, B, Zueger, B. J, Koestli, K, Erni, D, Schaffner, T, \& Banic, A. Weber H. P \& Frenz, M ((2002). Dye-Enhanced Laser Soldering of Cartilage and Blood Vessels. Laser Physics.12 (4): 635-640. Accessed 2013 March 5. Available: www.maik.ru/full/ lasphys/02/4/lasphys4_02pdf.Accessed 2013 March 3

[11] Khosroshahi, M. E, Nourbakhsh, M. S, Saremi, S, \& Tabatabaee, F. (2010). Characterization of Skin Tissue Soldering using Diode Laser and Indocyanine green: In Vitro Studies. Lasers in Med. Sci. Avaliable: link.springer.com/article/ 10.1007\%2Fs10103-009-0679-y. Accessed: 2013 March 3, 25(2), 207-212.

[12] Kirsch, A. J, Duckett, J. W, Snyder, H. M, Canning, D. A, Harshaw, D. W, \& Howard, P. Macarak E.J \& Zderic S.A ((1997). Skin Flap Closure by Dermal Laser Soldering: A Wound Healing Model Sutureless Hypospadies Repair. Pedia. Urology (Elsevier). Available: www.sciencedirect.com/science/article/pii/S0090429597002781.Accessed: 2013 March 3, 50(2), 263-272.

[13] Wadia, Y, Xie, H, \& Kajitani, M. (2000). Liver Repair and Hemorrhage Control by using Laser Soldering of Liquid Albumin in a porcine Model, Lasers Surg. Med., Available: http://www.ncbi.nlm.nih.gov/pubmed/11074508.Accessed : 2013 March 5, 27(4), 319-328.

[14] Xiong, Z, Zou, X, Wang, Y, \& Ding, H. (2010). Development and Error Compensation ofLlaser Soldering System. Assembly Automation, Available: http://dx.doi.org/ 10.1108/01445151011061109. Accessed 2013 Feb 13, 30 
[15] Renil S \& Hoppert B ((2011). Pyrometer Optimized Laser-based Selective Soldering Process; A Proven Process in an Industrial Manufacturing Environment. Avaliable : www.dilas.com/.../DILAS_Pyrometer\%20optimized\%20laser-...Accessed: 2013 Feb 22

[16] Cerda, E. (2000). Laser Soldering Applications for RF Shield Rework. Emerging technologies conference Proceedings., http://www.pcb-prototype.net/article/ Laser_Soldering.html,Accessed 2012 April 10.

[17] Basic Principle Soldering- Applications-Lasersystems (2012). http:// www.leister.com/en/grundprinzip-loeten.html,Accessed 2012 March 12.

[18] Strauss, R. (1998). SMT Soldering Handbook Newnes. 221.

[19] ((2012). http://www.leister.com/en/linienloeten.html,Accessed April 25.

[20] ((2012). http://www.plasticweldingstools.com/techtips/laser_solder_desolder.php,Accessed May 5.

[21] Vivari, J, \& Kasma, A. (2007). Laser Solder Reflow: A Process Solution. http:// www.nordson.com/.../solder/Nordson-EFD-Laser-Reflow-Soldering/,Accessed 2012 Feb. 20.

[22] Juddy, M, \& Brindley, K. (1999). Soldering in Electronics Assembly. Newnes, , 178-179.

[23] Strauss, R. (1998). SMT Soldering Handbook Newnes. , 219-220.

[24] Hecht, J. (1994). Understanding Lasers: An entry Level. IEEE.

[25] Unitek Miyachi Corporation (2003). Laser Welding Fundamentals. http:// www.unitekmiyachi.com,Accessed 2012 March 3.

[26] Qin, Yi. (2009). micro manufacturing Engineering \& Technology. 187.

[27] Ezeonu, S O. (2006). Introduction to Laser Technology. Rex Charles \& Patrick, 27.

[28] Tonshoff, H. K, Berndt, A, Sturmer, M, Golla, D, \& Schumacher, J. (1994). Diode Lasers for Material Processing. Journal de Physique, http://dx.doi.org/10.1051/ jp4:1994411, Accessed 2012 May 2.

[29] Visotek Inc, (2012). Diode Laser soldering. http://www.visotekinc.com/diode-laserapplications/diode-laser-soldering,Accessed 2012 April 3.

[30] Wolf Produktionssysteme (2012). Laser Soldering with High power Diode laser. http://www.orion-technology.fr/robot_laser/BrochureLaserSoldering.pdf,Accessed 2012 May 10.

[31] Hwang, J S. (2004). Solder technologies for Electronic packaging and Assembly. Haper C A, Electronic Materials and Process Handbook. McGraw-Hill, 5.

[32] Macapagal, R. (2003). Automated selective Soldering. http://www.empf.org/emphfasis/archive/1203automated.htm,Accessed 2012 June 15. 
[33] Zarrow, P, \& Klenke, B. (2003). Selective Soldering-The future is now: Opimize your Through-hole Soldering Process down to Pin Level. Circuit Assembly j. 14(4):16, Available: http://www.novaenginc.com/articles/columnselective\%soldering.pdf,Accessed 2012 June 12.

[34] Horn, W. (2009). Welding and Soldering with High Powered Diode Laser. http:// www.dilas.com/gdresources/downloads/.../DILAS_IIW_June09,Accessed 2012 April 12.

[35] Horn, W. (2007). High Power Diode Lasers for Industrial Applications. Laser Technik j, Available: onlinelibrary.wiley.com/doi/10.1002/latj.200790169/pdf , 4(3), 62-65.

[36] Brandner, M, Seibold, G, \& Chang, C. Dausinger F \& Hugel H ((2000). Soldering with Solid State and Diode Lasers: Energy Coupling, Temperature Rise, Process Window. J of laser Appl. Available: http://dx.doi.org/10.2351/1.1317551. accessed: 2013 March 8, 12(5), 194-199. 
